\title{
Numerical Solution of Coupled System of Nonlinear Partial Differential Equations Using Laplace-Adomian Decomposition Method
}

\author{
Mohamed S. M. Bahgat \\ Department of Mathematics, Faculty of Science, Minia University, Egypt \\ mohamed.bahagat@mu.edu.eg \\ Or \\ msmbahgat66@hotmail.com
}

\begin{abstract}
Aim of the paper is to investigate applications of Laplace Adomian Decomposition Method (LADM) on nonlinear physical problems. Some coupled system of non-linear partial differential equations (NLPDEs) are considered and solved numerically using LADM. The results obtained by LADM are compared with those obtained by standard and modified Adomian Decomposition Methods. The behavior of the numerical solution is shown through graphs. It is observed that LADM is an effective method with high accuracy with less number of components.
\end{abstract}

Mathematics Subject Classification: 45J05, 45B05, 45D05 and 45A05

Keywords: Laplace Adomian Decomposition Method; Adomian's Polynomial; Coupled partial differential equation.

\section{INTRODUCTION}

The partial differential equations (PDEs) have so many essential applications of science and engineering such as wave propagation, shallow water waves, fluid mechanics, thermodynamic, chemistry and micro electro mechanic system, etc. It is difficult to handle nonlinear part of these systems, although most of the scientists applied numerical methods to find the solutions of these systems that based on linearization, perturbed and discretizations. Debnath [1] applied the characteristics method and Logan [2] used the Rieman invariants method to handle systems of PDEs. Wazwaz [3] used the Adomian decomposition method (ADM) to handle the systems of PDEs. Laplace Decomposition Method (LDM) is free of any small or large parameters and has advantages over other approximation techniques like perturbation, LDM requires no discretization and linearization, therefore, results obtained by LDM are more efficient and realistic. This method has been used to obtain approximate solutions of a class of nonlinear ordinary and PDEs [4-7]. In this paper, we compute numerical solutions to nonlinear systems of PDEs by using LADM. The numerical solutions become easier and higher accuracy than the standard Adomian Decomposition Method (ADM).

\section{LADM for NONLINEAR COUPLED of PDEs}

Consider the general nonlinear coupled of PDEs written in an operators form (see [8])

$\left\{\begin{array}{l}L_{t}(u)+R_{1}(u)+M_{1}(u)+N_{1}(u, v)=f_{1}(x, t) \\ L_{t}(v)+R_{2}(v)+M_{2}(v)+N_{2}(u, v)=f_{2}(x, t)\end{array}\right.$

Subject to the initial conditions

$\left\{\begin{array}{l}u(x, 0)=g_{1}(x), 0 \leq x \leq \ell_{1} \\ v(x, 0)=g_{2}(x), 0 \leq x \leq \ell_{2}\end{array}\right.$

where $\ell_{1}$ and $\ell_{2}$ are real constants and the notations of $L_{t}=\frac{\partial}{\partial t}, R_{1}$ and $R_{2}$ symbolized the linear spatial differential operators, the notations $M_{1}, M_{2}, N_{1}$ and $N_{2}$ symbolized the nonlinear differential operators and $f_{1}(x, t), f_{2}(x, t)$ are given functions. The method consists of first applying the Laplace transform to both sides of equations in system (1) and then by using initial conditions, we have

$$
\left\{\begin{array}{l}
£\{u\}=\frac{g_{1}(x)}{s}+\frac{1}{s} £\left\{f_{1}(x, t)\right\}-\frac{1}{s}\left[£\left\{R_{1}(u)\right\}+£\left\{M_{1}(u)\right\}+£\left\{N_{1}(u, v)\right\}\right] \\
£\{v\}=\frac{g_{2}(x)}{s}+\frac{1}{s} £\left\{f_{2}(x, t)\right\}-\frac{1}{s}\left[£\left\{R_{2}(v)\right\}+£\left\{M_{2}(v)\right\}+£\left\{N_{2}(u, v)\right\}\right]
\end{array}\right.
$$

in the Laplace decomposition method we assume the solution is in an infinite series, given as follows 


$$
\left\{\begin{array}{l}
u(x, t)=\sum_{k=0}^{\infty} u_{k}(x, t), \\
v(x, t)=\sum_{k=0}^{\infty} v_{k}(x, t),
\end{array}\right.
$$

where $u_{k}(x, t)$ and $v_{k}(x, t)$ are to be recursively computed. Also the nonlinear terms $M_{1}, M_{2}, N_{1}$ and $N_{2}$ are decomposed as an infinite series of Adomian polynomials (see [9,10]),

$$
M_{1}(u)=\sum_{k=0}^{\infty} A_{n}, N_{1}(u, v)=\sum_{k=0}^{\infty} B_{n},
$$

For the nonlinear operators $M_{1}(\mathrm{u})$, and $N_{1}(\mathrm{u}, \mathrm{v})$ the Adomian's polynomials can be generated for all forms of nonlinearity. They are defined by the following relations

$$
\begin{aligned}
& A_{n}\left(u_{0}, u_{0}, \ldots, u_{n}\right)=\frac{1}{n !} \frac{d^{n}}{d \lambda^{n}}\left[M_{1}\left(\sum_{k=0}^{n} \lambda^{k} u_{k}(x, t)\right)\right]_{\lambda=0}, \\
& B_{n}\left(u_{0}, u_{0}, \ldots, u_{n} ; v_{0}, v_{0}, \ldots, v_{n}\right)=\frac{1}{n !} \frac{d^{n}}{d \lambda^{n}}\left[N_{1}\left(\sum_{k=0}^{n} \lambda^{k} u_{k}(x, t), \sum_{k=0}^{n} \lambda^{k} v_{k}(x, t)\right)\right]_{\lambda=0} .
\end{aligned}
$$

Substituting (4) and (6) into (3) and applying the linearity of the Laplace transform, we get the following recursively formula $\left\{\begin{array}{l}£\left\{u_{0}(x, t)\right\}=\frac{g_{1}(x)}{s}+\frac{1}{s} £\left\{f_{1}(x, t)\right\} \\ £\left\{v_{0}(x, t)\right\}=\frac{g_{2}(x)}{s}+\frac{1}{s} £\left\{f_{2}(x, t)\right\}\end{array}\right.$

and

$$
\begin{cases}£\left\{u_{k+1}(x, t)\right\}=-\frac{1}{s} £\left\{R_{1}\left(u_{k}(x, t)\right)+A_{k}+B_{k}\right\}, & k \geq 0 \\ £\left\{v_{k+1}(x, t)\right\}=-\frac{1}{s} £\left\{R_{2}\left(v_{k}(x, t)+C_{k}+D_{k}\right\},\right. & k \geq 0\end{cases}
$$

Applying the inverse Laplace transform, we can evaluate $u_{k}(x, t)$ and $v_{k}(x, t)$

\section{APPLICATIONS}

In order to verify numerically whether the proposed methodology leads to the accurate solutions, we evaluate LADM using the approximation for some examples of non-linear systems of PDEs. To show the efficiency of the present methods for our problems in comparison with the exact solution, we report the absolute error. The calculations in this paper have been done using the Maple 18 software. The results are listed in tables 1-2 and figures 1-4 below.

Example 1: Consider the coupled system of nonlinear PDE of the form [11]

$$
\left\{\begin{array}{l}
p_{t}+v_{x} w_{y}-v_{y} w_{x}=-p \\
v_{t}+w_{x} p_{y}+p_{x} w_{y}=v \\
w_{t}+p_{x} v_{y}+p_{y} v_{x}=w
\end{array}\right.
$$

with the following initial conditions 


$$
\left\{\begin{array}{l}
p(x, y, 0)=e^{x+y}, \\
v(x, y, 0)=e^{x-y} \\
w(x, y, 0)=e^{y-x} .
\end{array}\right.
$$

The exact solution of the system (9) is [11].

$$
\left\{\begin{array}{l}
p(x, \mathrm{y}, t)=e^{x+y-t} \\
v(x, y, t)=e^{x-y+t} \\
w(x, y, t)=e^{y-x+t}
\end{array}\right.
$$

Solution:_To solve the system of equations (9)-(10) by means of LADM, we construct a correctional functional which reads

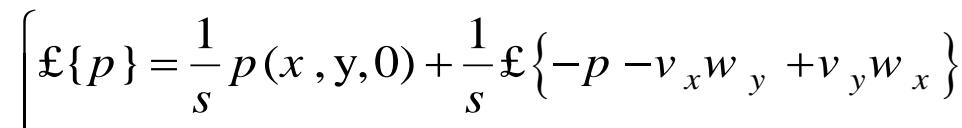

$$
\begin{aligned}
& \left\{\mathfrak{f}\{v\}=\frac{1}{s} v(x, \mathrm{y}, 0)+\frac{1}{s} £\left\{v-w_{x} p_{y}-p_{x} w_{y}\right\}\right. \\
& £\{\mathrm{w}\}=\frac{1}{s} \mathrm{w}(x, \mathrm{y}, 0)+\frac{1}{s} £\left\{w-p_{x} v_{y}-p_{y} v_{x}\right\}
\end{aligned}
$$

We can define the Adomian polynomials as follows

$$
\left\{\begin{array}{l}
A_{n}=\sum_{k=0}^{n}\left(\frac{\partial}{\partial x} v_{n-k}(x, y, t)\right)\left(\frac{\partial}{\partial y} w_{n-k}(x, y, t)\right), \\
B_{n}=\sum_{k=0}^{n}\left(\frac{\partial}{\partial y} v_{n-k}(x, y, t)\right)\left(\frac{\partial}{\partial x} w_{n-k}(x, y, t)\right), \\
C_{n}=\sum_{k=0}^{n}\left(\frac{\partial}{\partial x} w_{n-k}(x, y, t)\right)\left(\frac{\partial}{\partial y} p_{n-k}(x, y, t)\right), \\
E_{n}=\sum_{k=0}^{n}\left(\frac{\partial}{\partial x} p_{n-k}(x, y, t)\right)\left(\frac{\partial}{\partial y} w_{n-k}(x, y, t)\right), \\
F_{n}=\sum_{k=0}^{n}\left(\frac{\partial}{\partial x} p_{n-k}(x, y, t)\right)\left(\frac{\partial}{\partial y} v_{n-k}(x, y, t)\right), \\
G_{n}=\sum_{k=0}^{n}\left(\frac{\partial}{\partial y} p_{n-k}(x, y, t)\right)\left(\frac{\partial}{\partial x} v_{n-k}(x, y, t)\right) .
\end{array}\right.
$$

We define an iterative scheme 


$$
\left\{\begin{array}{l}
£\left\{p_{0}(x, y, t)\right\}=\frac{1}{s} e^{x+y} \\
£\left\{v_{0}(x, y, t)\right\}=\frac{1}{s} e^{x-y} \\
£\left\{w_{0}(x, y, t)\right\}=\frac{1}{s} e^{y-x} \\
£\left\{p_{n+1}(x, y, t)\right\}=\frac{1}{s} £\left\{-p_{n}(x, y, t)-A_{n}+B_{n}\right\}, \quad n \geq 0 \\
£\left\{v_{n+1}(x, y, t)\right\}=\frac{1}{s} £\left\{v_{n}(x, y, t)-C_{n}-E_{n}\right\}, \quad n \geq 0 \\
£\left\{w_{n+1}(x, y, t)\right\}=\frac{1}{s} £\left\{w_{n}(x, y, t)-F_{n}-G_{n}\right\}, \quad n \geq 0
\end{array}\right.
$$

the Adomian's polynomials $A_{n}, B_{n}, C_{n}, E_{n}, F_{n}$ and $G_{n}$ are generated according to (6), we can give the first few Adomian's polynomials as follows

$$
\begin{aligned}
& A_{0}=1, A_{1}=t^{2}+1, A_{2}=\frac{1}{4} t^{4}+t^{2}+1, A_{3}=\frac{1}{36} t^{6}+\frac{1}{4} t^{4}+t^{2}+1 \\
& B_{0}=1, B_{1}=t^{2}+1, B_{2}=\frac{1}{4} t^{4}+t^{2}+1, B_{3}=\frac{1}{36} t^{6}+\frac{1}{4} t^{4}+t^{2}+1 \\
& C_{0}=-e^{2 y}, C_{1}=e^{2 y}\left(t^{2}-1\right), C_{2}=-\frac{1}{4} e^{2 y}\left(t^{4}-4 t^{2}+4\right), \\
& C_{3}=\frac{1}{36} e^{2 y}\left(t^{6}-9 t^{4}+36 t^{2}-36\right), E_{0}=e^{2 y}, E_{1}=-e^{2 y}\left(t^{2}-1\right), \\
& E_{2}=\frac{1}{4} e^{2 y}\left(t^{4}-4 t^{2}+4\right), E_{3}=-\frac{1}{36} e^{2 y}\left(t^{6}-9 t^{4}+36 t^{2}-36\right), \\
& F_{0}=-e^{2 x}, F_{1}=e^{2 x}\left(t^{2}-1\right), F_{2}=\frac{1}{4} e^{2 y}\left(t^{4}-4 t^{2}+4\right), \\
& F_{3}=\frac{1}{36} e^{2 x}\left(t^{6}-9 t^{4}+36 t^{2}-36\right), G_{0}=e^{2 x}, G_{1}=-e^{2 x}\left(t^{2}-1\right), \\
& G_{2}=\frac{1}{4} e^{2 x}\left(t^{4}-4 t^{2}+4\right), G_{3}=-\frac{1}{36} e^{2 x}\left(t^{6}-9 t^{4}+36 t^{2}-36\right),
\end{aligned}
$$

Applying the inverse Laplace transform, finally, According to (11) the $0^{\text {th }}$ components

$p_{0}(x, y, t)=\mathrm{e}^{x+y}, v_{0}(x, y, t)=\mathrm{e}^{x-y}$ and $w_{0}(x, y, t)=\mathrm{e}^{y-x}$ written as follows:

$p_{0}(x, y, t)=\mathrm{e}^{x+y}, v_{0}(x, y, t)=\mathrm{e}^{x-y}, w_{0}(x, y, t)=\mathrm{e}^{y-x}$, where, $k \geq 0$. So, we get the following components:

$$
\begin{aligned}
& p_{1}(x, y, t)=\mathrm{e}^{x+y} \mathrm{t}, v_{1}(x, y, t)=\mathrm{e}^{x-y} \mathrm{t}, w_{1}(x, y, t)=\mathrm{e}^{y-x} t, \\
& p_{2}(x, y, t)=\frac{1}{2} \mathrm{e}^{x+y} \mathrm{t}^{2}, v_{2}(x, y, t)=\frac{1}{2} \mathrm{e}^{x-y} \mathrm{t}^{2}, w_{2}(x, y, t)=\frac{1}{2} \mathrm{e}^{y-x} t^{2}, \\
& p_{3}(x, y, t)=-\frac{1}{6} \mathrm{e}^{x+y} \mathrm{t}^{3}, v_{3}(x, y, t)=\frac{1}{6} \mathrm{e}^{x-y} \mathrm{t}^{3}, w_{3}(x, y, t)=\frac{1}{6} \mathrm{e}^{y-x} t^{3}
\end{aligned}
$$


$p_{30}(x, y, t)=\frac{1}{265252859812191058636308480000000} \mathrm{e}^{x+y} \mathrm{t}^{30}$,

$v_{30}(x, y, t)=\frac{1}{265252859812191058636308480000000} \mathrm{e}^{x-y} \mathrm{t}^{30}$,

$w_{0}(x, y, t)=\frac{1}{265252859812191058636308480000000} \mathrm{e}^{y-x} \mathrm{t}^{30}$,

Similarly, we can also find other components, and the approximate solution for calculating $50^{\text {th }}$. Using (4), the series solutions are therefore given by

$$
\begin{aligned}
& p(x, y, t)=-\frac{1}{120} e^{x+y}\left(t^{5}-5 t^{4}+20 t^{3}-60 t^{2}+120 t-120+\cdots\right), \\
& v(x, y, t)=\frac{1}{120} e^{x-y}\left(t^{5}+5 t^{4}+20 t^{3}+60 t^{2}+120 t+120+\cdots\right), \\
& v(x, y, t)=\frac{1}{120} e^{y-x}\left(t^{5}+5 t^{4}+20 t^{3}+60 t^{2}+120 t+120+\cdots\right) .
\end{aligned}
$$

By using the Taylor expansion for $e^{t}$ and $e^{-t}$ we can find the exact solutions. The numerical behaviors of approximate solutions of LADM of $p(x, y, t), v(x, y, t)$ and $w(x, y, t)$ with different values of time are compared with the exact solution are shown through figure1(a)-(c)

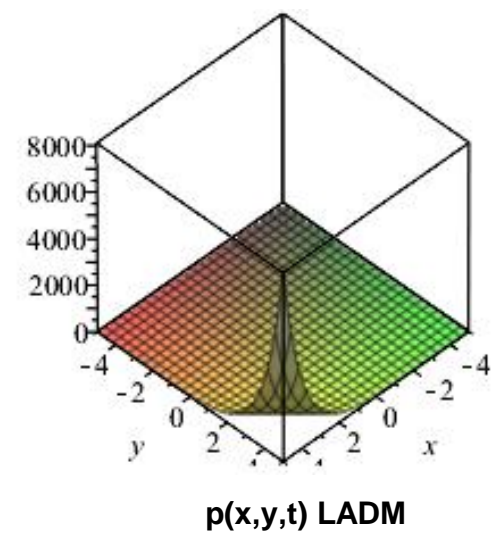

(a)

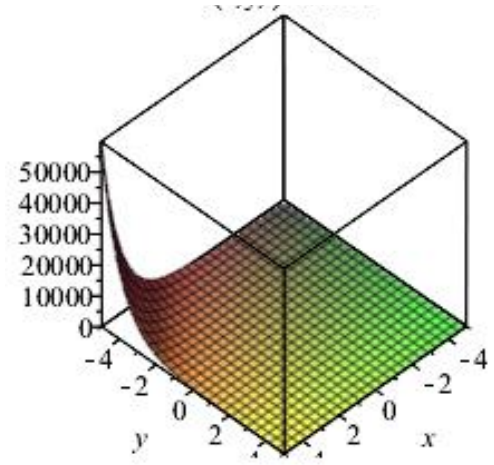

$v(x, y, t)$ LADM

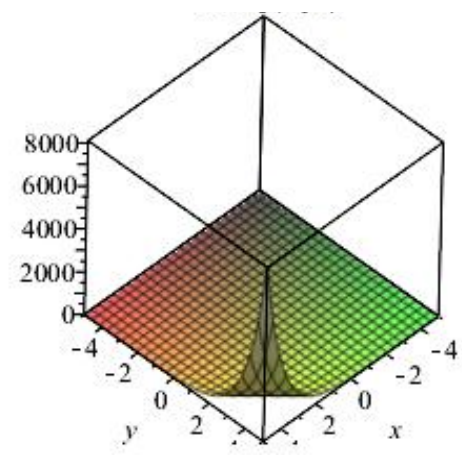

$p(x, y, t)$ exact

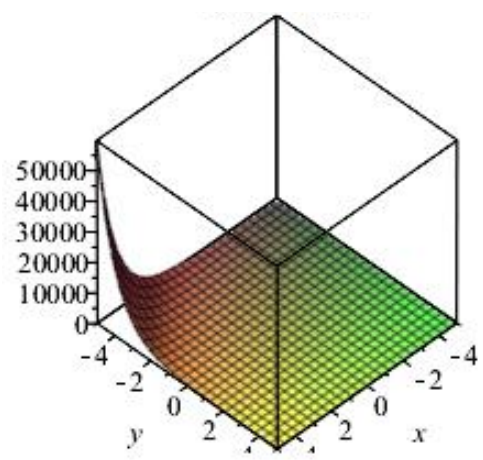

$v(x, y, t)$ exact

(b) 


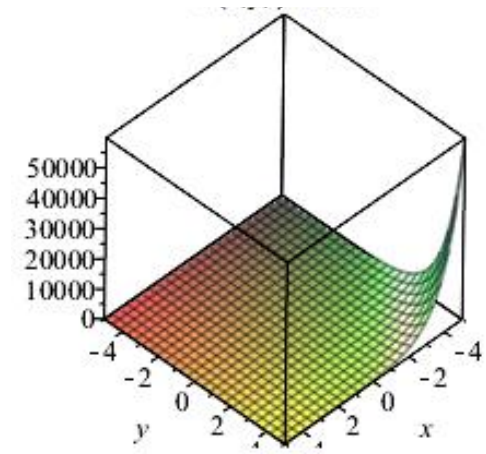

$w(x, y, t)$ LADM

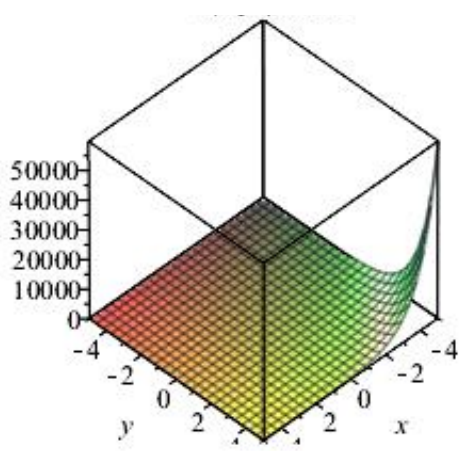

$w(x, y, t)$ exact

(c)

Figure 1. (a) Exact and numerical solution of $p(x, y, t),-5 \leq x \leq 5,-5 \leq y \leq 5$ for different values of t; (b) Exact and numerical solution of $v(x, \mathrm{y}, t),-5 \leq x \leq 5,-5 \leq y \leq 5$ for different values of $\mathrm{t}$; (c) Exact and numerical solution of $w(x, \mathrm{y}, t)$, $-5 \leq x \leq 5,-5 \leq y \leq 5$ for different values of $\mathrm{t}$

Example 2: The coupled Burgers system

$u_{t}-u_{x x}-2 u u_{x}+(u v)_{x}=0$

$v_{t}-v_{x x}-2 v v_{x}+(u v)_{x}=0$

where $q_{s}=\frac{\partial}{\partial s} \mathrm{q}(x, t), q_{s s}=\frac{\partial^{2}}{\partial s^{2}} q(x, t)$, with initial conditions $u(x, 0)=\sin x, \quad v(x, 0)=\sin x$. The exact solution of the system (14) is $u(x, \mathrm{t})=v(x, t)=\sin x e^{-t}$ (see [11] and [12] ).

Solution: To solve the system of equation (14) by means of LADM , we construct a correctional functional which reads

$£\{u\}=\frac{1}{s} u(x, 0)+\frac{1}{s} £\left\{u_{x x}+2 u u_{x}-(u v)_{x}\right\}$

$£\{v\}=\frac{1}{s} v(x, 0)+\frac{1}{s} £\left\{v_{x x}+2 v v_{x}-(u v)_{x}\right\}$

We can define the Adomian polynomials as follows

$\left\{\begin{array}{l}A_{n}=\sum_{k=0}^{n} \frac{\partial^{2}}{\partial x^{2}} u_{k}(x, t), \quad B_{n}=2 \sum_{k=0}^{n} u_{k}(x, t) \frac{\partial}{\partial x} u_{n-k}(x, t), \\ C_{n}=\sum_{k=0}^{n} \frac{\partial}{\partial x}\left(u_{n-k}(x, t) v_{n-k}(x, t)\right), \quad F_{n}=\sum_{k=0}^{n} \frac{\partial^{2}}{\partial x^{2}} v_{k}(x, t) \\ E_{n}=2 \sum_{k=0}^{n} v_{k}(x, t) \frac{\partial}{\partial x} v_{n-k}(x, t),\end{array}\right.$

We define an iterative scheme

$\left\{\begin{array}{l}£\left\{u_{0}(x, t)\right\}=\frac{1}{s} \sin x \\ £\left\{v_{0}(x, t)\right\}=\frac{1}{s} \sin x \\ £\left\{u_{n+1}(x, t)\right\}=\frac{1}{s} £\left\{A_{n}+B_{n}-C_{n}\right\}, \quad n \geq 0 \\ £\left\{v_{n+1}(x, t)\right\}=\frac{1}{s} £\left\{F_{n}+E_{n}-C_{n}\right\}, \quad n \geq 0 .\end{array}\right.$

Applying the inverse Laplace transform, finally, the Adomian's polynomials $A_{n}, B_{n}, C_{n}, E_{n}$ and $F_{n}$ are generated according to (6), we can give the first few components of the Adomian's polynomials respectively as follows 


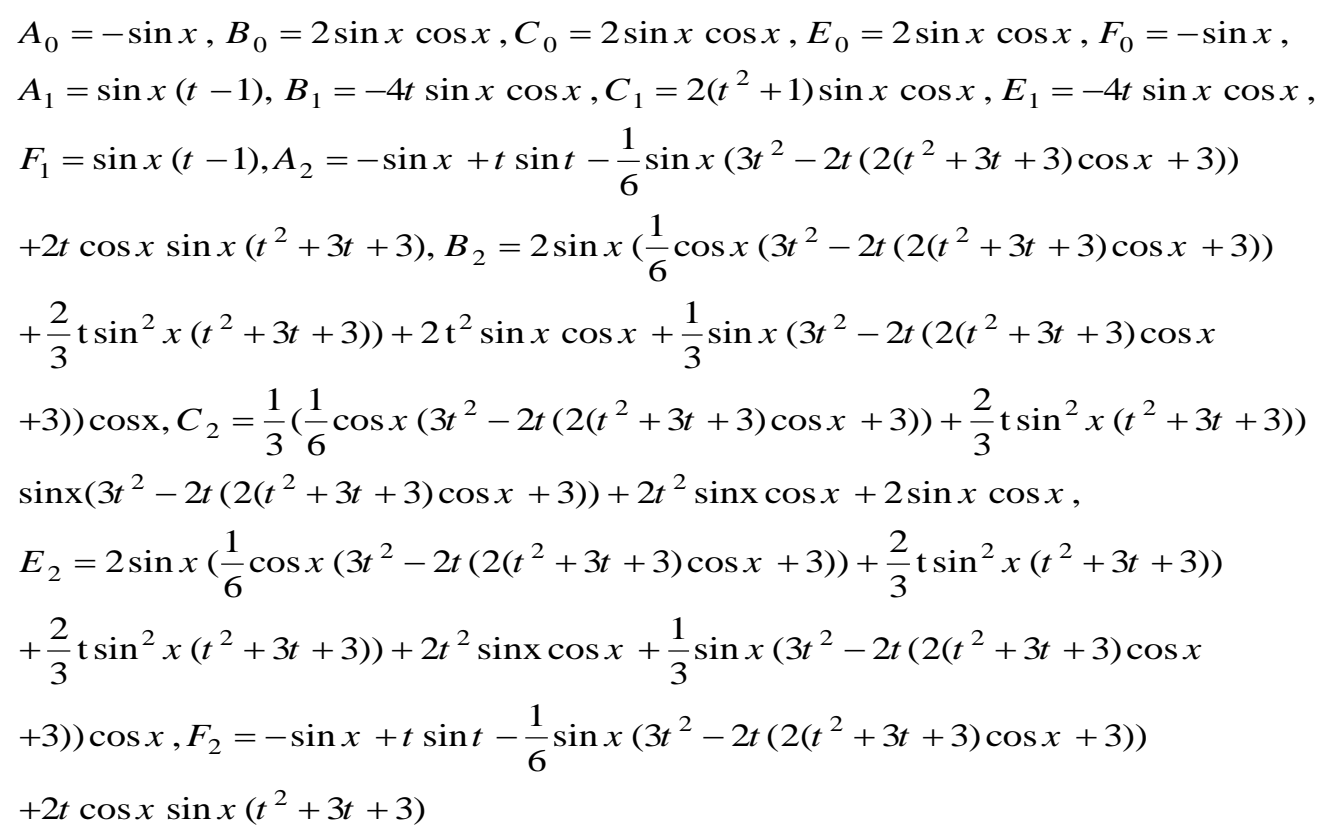

According to (7), the $0^{t h}$ components $u_{0}(x, t)$ and $v_{0}(x, t)$ written as follows: $u_{0}(x, t):=\sin (x)$ $v_{0}(x, t):=\sin (x)$, where, $n \geq 0$. So, we get the following components:

$$
\begin{aligned}
& u_{1}(x, t)=-t \sin x, v_{1}(x, t)=-t \sin x \\
& u_{2}(x, t)=\frac{1}{6} \sin x\left(3 t^{2}-2 t\left(2\left(t^{2}+3 t+3\right) \cos x+3\right)\right) \\
& v_{2}(x, t)=\frac{1}{6} \sin x\left(3 t^{2}-2 t\left(2\left(t^{2}+3 t+3\right) \cos x+3\right)\right) \\
& u_{3}(x, t)=\frac{1}{630} \mathrm{t}^{2} \sin ^{3} x\left(1260-70 t^{4}-84 t^{3}+525 t^{2}+40 t\left(\left(2 t^{4}+14 t^{3}\right.\right.\right. \\
& \left.\left.\left.+42 t^{2}+63 t+42\right) \cos +42\right)\right)+\frac{1}{630}\left(-630-21 \cos \left(3 \mathrm{t}^{4}-35 t^{3}-80 t^{2}-60 t+60\right)\right. \\
& -5 t^{2}\left(8 \cos ^{3} x\left(2 t^{4}+14 t^{3}+42 t^{2}+63 t+42\right)+21\right)+14 t\left(\cos ^{2} x(5 t+6)\right. \\
& \left.\left.\left(2 \mathrm{t}^{3}-15 t-30\right)+45\right)\right) \mathrm{t} \sin x \\
& v_{3}(x, t)=\frac{1}{630} \mathrm{t}^{2} \sin { }^{3} x\left(1260-70 t^{4}-84 t^{3}+525 t^{2}+40 t\left(\left(2 t^{4}+14 t^{3}\right.\right.\right. \\
& \left.\left.\left.+42 t^{2}+63 t+42\right) \cos +42\right)\right)+\frac{1}{630}\left(-630-21 \cos \left(3 \mathrm{t}^{4}-35 t^{3}-80 t^{2}-60 t+60\right)\right. \\
& -5 t^{2}\left(8 \cos ^{3} x\left(2 t^{4}+14 t^{3}+42 t^{2}+63 t+42\right)+21\right)+14 t\left(\cos ^{2} x(5 t+6)\right. \\
& \left.\left.\left(2 \mathrm{t}^{3}-15 t-30\right)+45\right)\right) \mathrm{t} \sin x
\end{aligned}
$$

Similarly, we can also find other components, and the approximate solution for calculating $5^{\text {th }}$. Using (4), the series solutions are therefore given by

$u(x, t)=\sin x-t \sin x+\frac{1}{6} \sin x\left(3 t^{2}-2 t\left(2\left(t^{2}+3 t+3\right) \cos x+3+\cdots\right)\right)$
$v(x, t)=\sin x-t \sin x+\frac{1}{6} \sin x\left(3 t^{2}-2 t\left(2\left(t^{2}+3 t+3\right) \cos x+3+\cdots\right)\right)$

and

Figures 2. (a) and (b) show the exact and numerical solution of system (14) with $10^{\text {th }}$ terms by LADM. 


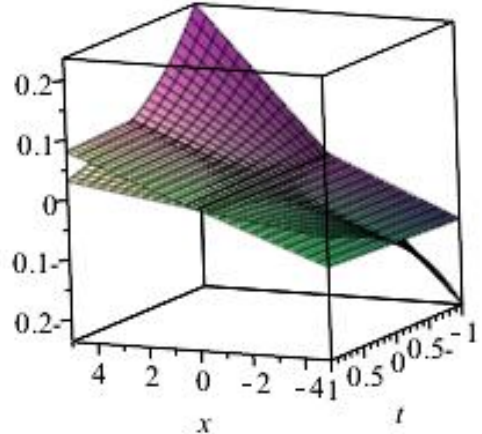

LADM and exact for $u(x, t)$

(a)

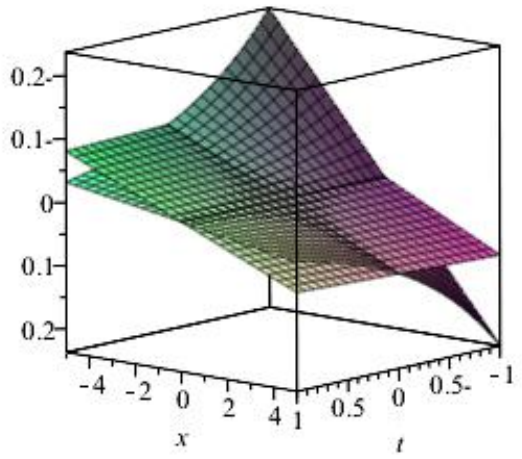

LADM and exact for $v(x, t)$

(b)

Figure 2. (a) show the exact and LADM numerical solution $u(x, t)$ of example $2,-5 \leq x \leq 5,-1 \leq t \leq 1$; (b) show the exact and LADM numerical solution $v(x, t)$ of example $2,-5 \leq x \leq 5,-1 \leq t \leq 1$.

Example 3: we consider the nonlinear system [13]

$u_{t}=u u_{x}+v u_{y}$
$v_{t}=v v_{y}+u v_{x}$,

with the initial conditions $u(x, \mathrm{y}, 0)=x+y ; \quad \mathrm{v}(\mathrm{x}, \mathrm{y}, 0)=x+y, \quad 0 \leq x, y \leq 1$.

The exact solution given as $u(x, \mathrm{y}, t)=\frac{x+y}{1-2 t} ; \quad \mathrm{v}(\mathrm{x}, \mathrm{y}, t)=\frac{x+y}{1-2 t}, \quad 0 \leq t \leq T$.

Solution: Taking the Laplace transform on both sides of Eqs. (10) then, by using the differentiation property of Laplace transform and initial conditions, as the same procedure in the above example when we using equations (9)-(10) for the system (10) and according to (3) we have the following $0^{\text {th }}$ components

$u_{0}(x, y, t):=x+y ; v_{0}(x, y, t):=x+y$, and the recursive relation can be written as follows:

$u_{1}(x, y, t):=2(x+y) t ; v_{1}(x, y, t):=2(x+y) t ; u_{2}(x, y, t):=4(x+y) t^{2}$

$v_{2}(x, y, t):=4(x+y) t^{2} ; u_{3}(x, y, t):=8(x+y) t^{3} ; v_{3}(x, y, t):=8(x+y) t^{3}$;

$u_{4}(x, y, t):=16(x+y) t^{4} ; v_{4}(x, y, t):=16(x+y) t^{4}$

$u_{20}(x, y, t):=1048576(x+y) t^{20} ; v_{20}(x, y, t):=1048576(x+y) t^{20}$

Similarly, we can also find other components, and the approximate solution for calculating more $20^{\text {th }}$. Using (3), the series solutions are therefore given by

$$
\begin{aligned}
u(x, \mathrm{y}, t)=v(x, \mathrm{y}, \mathrm{t}) & =(\mathrm{x}+\mathrm{y})+2(\mathrm{x}+\mathrm{y}) \mathrm{t}+4(\mathrm{x}+\mathrm{y}) \mathrm{t}^{2}+8(\mathrm{x}+\mathrm{y}) \mathrm{t}^{3}+16(\mathrm{x}+\mathrm{y}) \mathrm{t}^{4}+\cdots \\
= & (x+y)\left[1+2 t+4 t^{2}+8 t^{3}+16 t^{4}+\cdots\right] \\
& =(x+y)(1-2 t)^{-1}=\frac{x+y}{1-2 t}
\end{aligned}
$$

that converges to the exact solutions $u(x, \mathrm{y}, t)=\frac{x+y}{1-2 t} ; \quad v(x, y, t)=\frac{x+y}{1-2 t}$,

and

Figure 3 ensure that the previous obtained results of system (18) with $30^{\text {th }}$ terms by LADM it converges to the exact solutions. Table 1 show the absolute error between the exact solution and the results obtained from the LADM solution 
and comparison with the results obtained by the standard algorithm for Adomian's polynomials ADM and modified ADM solution of system of equation (18) (see [14] and [16]).

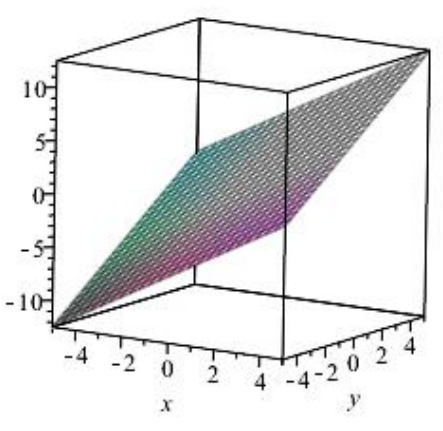

$\mathrm{u}(\mathrm{x}, \mathrm{y}, \mathrm{t})$ LADM

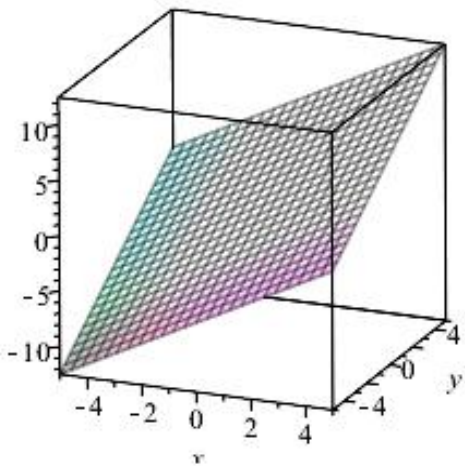

$v(x, y, t))$ LADM

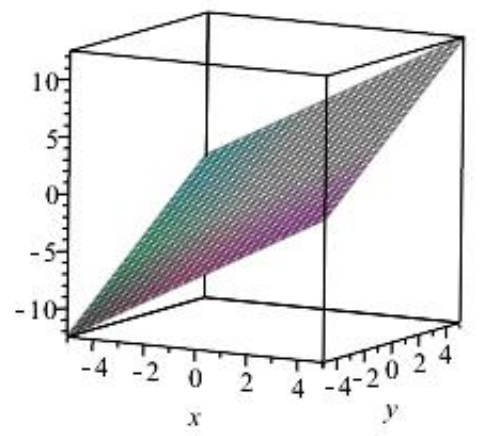

$u(x, y, t)$ and $v(x, y, t)$ exact

Figures 3. show the exact and LADM numerical solutions of $u(x, t)$ and $v(x, t)$ for example $3,-5 \leq x \leq 5,-1 \leq y \leq 1$; $\mathrm{t}=0.1$.

Table 1: The numerical results in comparison with the analytical solutions for various values of $x, y$ and $t$ for example 3

\begin{tabular}{|c|c|c|c|c|c|c|}
\hline \multirow[b]{2}{*}{$t$} & \multirow[b]{2}{*}{$y$} & \multirow[b]{2}{*}{$x$} & \multirow[b]{2}{*}{$\operatorname{Exact}(u)=\operatorname{Exact}(v)$} & \multicolumn{3}{|c|}{ The absolute error } \\
\hline & & & & LADM & AMD [16] & $\begin{array}{l}\text { Modified } \\
\text { AMD[14] }\end{array}$ \\
\hline \multirow{5}{*}{0.1} & \multirow{2}{*}{0.125} & 0.125 & 0.312500000 & $6.55 e-016$ & $3.2000 \mathrm{e}-008$ & 3. $0000 \mathrm{e}-010$ \\
\hline & & 0.785 & 1.137500000 & $2.39 \mathrm{e}-015$ & $1.1600 \mathrm{e}-007$ & $0.0000 e+000$ \\
\hline & 0.500 & 0.500 & 1.250000000 & $2.62 \mathrm{e}-015$ & $1.2800 \mathrm{e}-007$ & $0.0000 \mathrm{e}+000$ \\
\hline & \multirow{2}{*}{0.875} & 0.125 & 1.250000000 & $2.62 \mathrm{e}-015$ & $1.2800 \mathrm{e}-007$ & $0.0000 e+000$ \\
\hline & & 0.785 & 2.075000000 & $4.35 e-015$ & $2.1200 \mathrm{e}-007$ & $0.0000 \mathrm{e}+000$ \\
\hline \multirow{5}{*}{0.2} & \multirow{2}{*}{0.125} & 0.125 & 0.416666667 & $1.83 e-009$ & $4.3690 \mathrm{e}-005$ & 4. $2700 \mathrm{e}-008$ \\
\hline & & 0.785 & 1.516666667 & $6.67 e-009$ & $1.5903 e-004$ & 1. $5900 \mathrm{e}-007$ \\
\hline & 0.500 & 0.500 & 1.666666667 & $7.33 e-009$ & $1.7476 \mathrm{e}-004$ & 1. $6600 \mathrm{e}-007$ \\
\hline & \multirow{2}{*}{0.875} & 0.125 & 1.666666667 & $7.33 e-009$ & $1.7476 \mathrm{e}-004$ & 1. $6600 \mathrm{e}-007$ \\
\hline & & 0.785 & 2.766666667 & $1.22 \mathrm{e}-008$ & $2.9010 \mathrm{e}-004$ & 2. $8600 \mathrm{e}-007$ \\
\hline \multirow{5}{*}{0.3} & \multirow{2}{*}{0.125} & 0.125 & 0.625000000 & $1.37 e-005$ & $3.7791 \mathrm{e}-003$ & 1. $4145 \mathrm{e}-005$ \\
\hline & & 0.785 & 2.275000000 & $4.99 \mathrm{e}-005$ & $1.3756 \mathrm{e}-002$ & 5. $1480 \mathrm{e}-005$ \\
\hline & 0.500 & 0.500 & 2.500000000 & $5.48 e-005$ & $1.5116 \mathrm{e}-002$ & $5.6574 \mathrm{e}-005$ \\
\hline & \multirow{2}{*}{0.875} & 0.125 & 2.500000000 & $5.48 e-005$ & $1.5116 \mathrm{e}-002$ & 5. $6574 \mathrm{e}-005$ \\
\hline & & 0.785 & 4.150000000 & $9.10 \mathrm{e}-005$ & $2.5093 e-002$ & 9. $3897 e-005$ \\
\hline
\end{tabular}


We note from the above results, the absolute error obtained by the proposed algorithm LADM as compared with the absolute error of the standard algorithm for Adomian's polynomials ADM and modified ADM given results more accurate.

Example 4: The mathematical models on many phenomena in applied sciences lead to non-linear PDEs such as the homogeneous form of the system of two dimensional Burger's equations which is proposed as mathematical model of free turbulence $[15,16]$

$$
\left\{\begin{array}{l}
u_{t}+u u_{x}+v u_{y}=\frac{1}{R}\left(u_{x x}+u_{y y}\right), \\
v_{t}+v v_{y}+u v_{x}=\frac{1}{R}\left(v_{x x}+v_{y y}\right),
\end{array}\right.
$$

subject to the initial conditions

$$
\left\{\begin{array}{l}
u(x, \mathrm{y}, 0)=\frac{3}{4}-\frac{1}{4\left(1+e^{R(y-x) / 8}\right)}, \\
u(x, \mathrm{y}, 0)=\frac{3}{4}-\frac{1}{4\left(1+e^{R(y-x) / 8}\right)},
\end{array}\right.
$$

the exact solution [8]:

$$
\left\{\begin{array}{l}
u(x, \mathrm{y}, t)=\frac{3}{4}-\frac{1}{4\left(1+e^{R(-\mathrm{t}+4 y-4 x) / 32}\right)}, \\
v(x, \mathrm{y}, t)=\frac{3}{4}-\frac{1}{4\left(1+e^{R(-\mathrm{t}+4 y-4 x) / 32}\right)},
\end{array}\right.
$$

where R is Reynolds number. As the same procedure in the above examples when we use (3)-(6) for the system (19)-(20) and according to $(7)-(8)$, we have the following $0^{\text {th }}$ components:

$$
\left\{\begin{array}{l}
u_{0}(x, \mathrm{y}, t)=\left(\frac{3}{4}-\frac{1}{4\left(1+e^{R(y-x) / 8}\right)}\right), \\
v_{0}(x, \mathrm{y}, t)=\left(\frac{3}{4}-\frac{1}{4\left(1+e^{R(y-x) / 8}\right)}\right),
\end{array}\right.
$$

and the recursive relation can be written as follows:

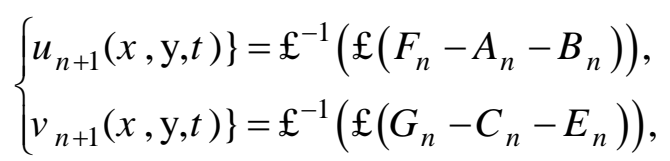

where $n \geq 0$ and the Adomian polynomials $A_{n}, B_{n}, C_{n}, E_{n}, F_{n}$ and $G_{n}$ are defind as follows

$A_{n}:=\sum_{k=0}^{\mathrm{n}} u_{k}(x, y, t) \cdot\left(\frac{\partial}{\partial x}\left(u_{(\mathrm{n}-k)}(x, y, t)\right)\right)$; 


$$
\begin{aligned}
& B_{n}:=\sum_{k=0}^{n} v_{k}(x, y, t) \cdot\left(\frac{\partial}{\partial y}\left(u_{(\mathrm{n}-k)}(x, y, t)\right)\right) ; \\
& C_{n}:=\sum_{k=0}^{n} v_{k}(x, y, t) \cdot\left(\frac{\partial}{\partial y}\left(v_{(\mathrm{n}-k)}(x, y, t)\right)\right) \\
& E_{n}:=\sum_{k=0}^{\mathrm{n}} u_{k}(x, y, t) \cdot\left(\frac{\partial}{\partial x}\left(v_{(\mathrm{n}-k)}(x, y, t)\right)\right) ; \\
& F_{n}:=\frac{1}{R} \sum_{k=0}^{\mathrm{n}}\left(\frac{\partial^{2}}{\partial x^{2}}\left(u_{k}(x, y, t)\right)+\frac{\partial^{2}}{\partial y^{2}}\left(u_{k}(x, y, t)\right)\right) \\
& G_{n}:=\frac{1}{R} \sum_{k=0}^{\mathrm{n}}\left(\frac{\partial^{2}}{\partial x^{2}}\left(v_{k}(x, y, t)\right)+\frac{\partial^{2}}{\partial y^{2}}\left(v_{k}(x, y, t)\right)\right) .
\end{aligned}
$$

We can give the first few Adomian's polynomials of respectively to get the following components:

$$
A_{0}:=-\frac{1}{128} \frac{\left(2+3 \mathrm{e}^{-\frac{1}{8} R(-y+x)}\right) R \mathrm{e}^{-\frac{1}{8} R(-y+x)}}{\left(1+\mathrm{e}^{-\frac{1}{8} R(-y+x)}\right)^{3}}
$$$$
A_{1}:=-\frac{1}{4096} \frac{R^{2} \mathrm{e}^{-\frac{1}{8} R(-y+x)} t\left(3 \mathrm{e}^{-\frac{1}{4} R(-y+x)}-2 \mathrm{e}^{-\frac{1}{8} R(-y+x)}-2\right)}{\left(1+\mathrm{e}^{-\frac{1}{8} R(-y+x)}\right)^{4}}
$$$$
B_{0}:=\frac{1}{128} \frac{R \mathrm{e}^{-\frac{1}{8} R(-y+x)}\left(4+3 \mathrm{e}^{-\frac{1}{8} R(-y+x)}\right)}{\left(1+\mathrm{e}^{-\frac{1}{8} R(-y+x)}\right)^{3}}
$$$$
B_{1}:=\frac{1}{4096} \frac{R^{2} \mathrm{e}^{-\frac{1}{8} R(-y+x)} t\left(3 \mathrm{e}^{-\frac{1}{4} R(-y+x)}+2 \mathrm{e}^{-\frac{1}{8} R(-y+x)}-4\right)}{\left(1+\mathrm{e}^{-\frac{1}{8} R(-y+x)}\right)^{4}}
$$$$
C_{0}:=-\frac{1}{128} \frac{R \mathrm{e}^{-\frac{1}{8} R(-y+x)}\left(4+3 \mathrm{e}^{-\frac{1}{8} R(-y+x)}\right)}{\left(1+\mathrm{e}^{-\frac{1}{8} R(-y+x)}\right)^{3}}
$$$$
C_{1}:=-\frac{1}{4096} \frac{R^{2} \mathrm{e}^{-\frac{1}{8} R(-y+x)} t\left(3 \mathrm{e}^{-\frac{1}{4} R(-y+x)}+2 \mathrm{e}^{-\frac{1}{8} R(-y+x)}-4\right)}{\left(1+\mathrm{e}^{-\frac{1}{8} R(-y+x)}\right)^{4}}
$$ 
$v_{2}(x, y, t):=\frac{1}{8192} \frac{R \mathrm{e}^{-\frac{1}{8} R(-y+x)}\left(\mathrm{e}^{-\frac{1}{8} R(-y+x)}-1\right) t(R t+64)}{\left(1+\mathrm{e}^{-\frac{1}{8} R(-y+x)}\right)^{3}}$.

Similarly, we can also find other components, and Using (3), the series solutions are therefore given by

$u(x, y, t):=-\frac{1}{128} \frac{R \mathrm{e}^{-\frac{1}{8} R(-y+x)} t-96 \mathrm{e}^{-\frac{1}{4} R(-y+x)}-160 \mathrm{e}^{-\frac{1}{8} R(-y+x)}-64}{\left(1+\mathrm{e}^{-\frac{1}{8} R(-y+x)}\right)^{2}}$

$v(x, y, t):=\frac{1}{128} \frac{R \mathrm{e}^{-\frac{1}{8} R(-y+x)} t+96 \mathrm{e}^{-\frac{1}{4} R(-y+x)}+224 \mathrm{e}^{-\frac{1}{8} R(-y+x)}+128}{\left(1+\mathrm{e}^{-\frac{1}{8} R(-y+x)}\right)^{2}}$

and

Figure 4. (a)-(d) show the exact and numerical solution of system (19) with $3^{\text {th }}$ terms by LADM also table 2 show the absolute error between the exact solution and the results obtained from the LADM solution and comparison with the results obtained by the standard algorithm ADM (see [16]).

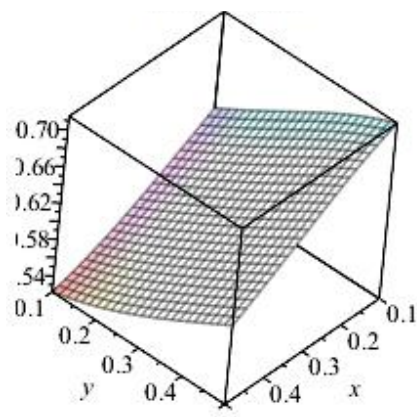

$u(x, y, t)$ LADM

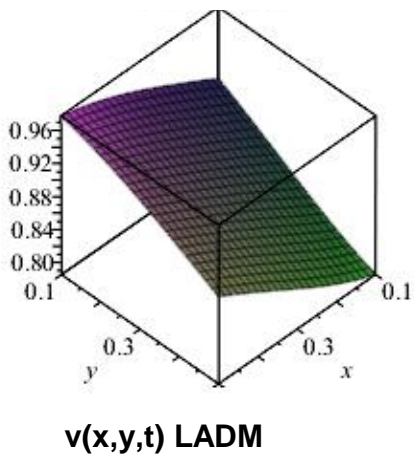

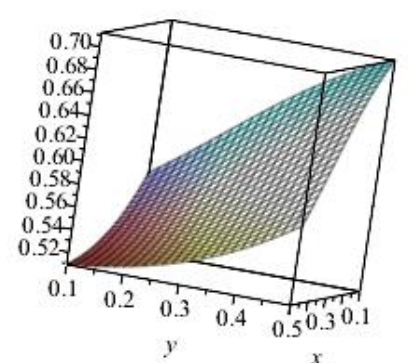

$u(x, y, t)$ exact

(a)

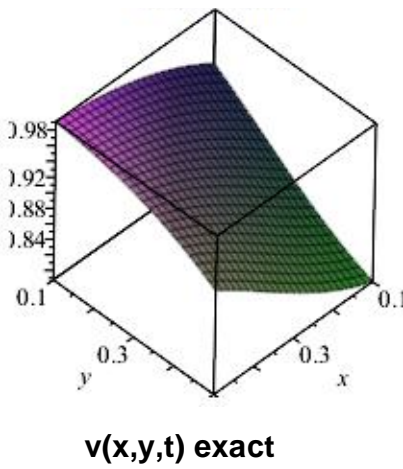

(b)

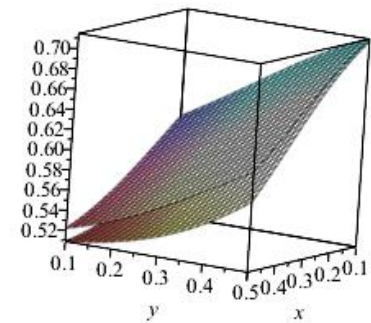

LADM and exact for $u(x, y, t)$

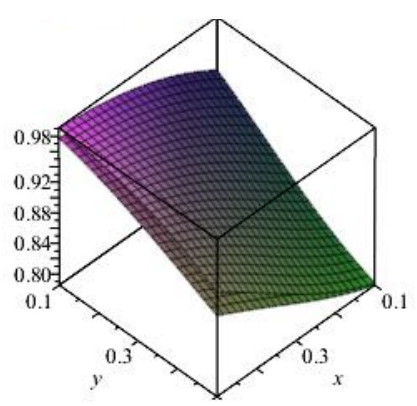

LADM and exact for $\mathbf{v}(\mathbf{x}, \mathbf{y}, \mathbf{t})$

Figures 4. (a) and (b) show the exact and LADM of $u(x, y, t)$ and $v(x, y, t)$ for example $4,0.1 \leq x \leq 0.5 ; 0.1 \leq y \leq 0.5$; $\mathrm{t}=0.2 ; \mathrm{R}=50$ 


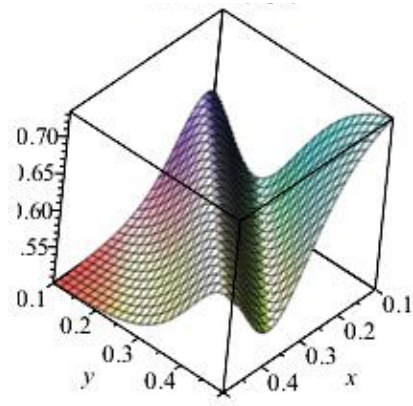

$u(x, y, t) L A D M$

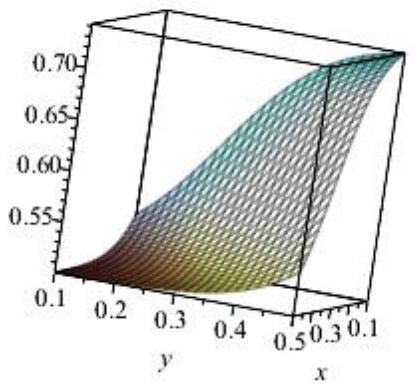

$u(x, y, t)$ exact

(c)

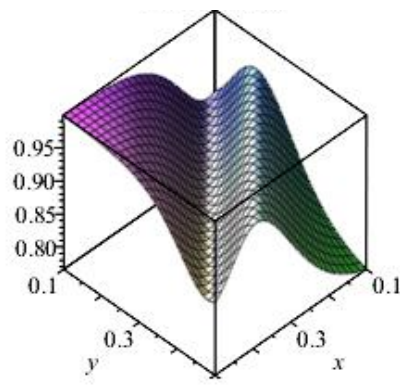

$v(x, y, t)$ LADM

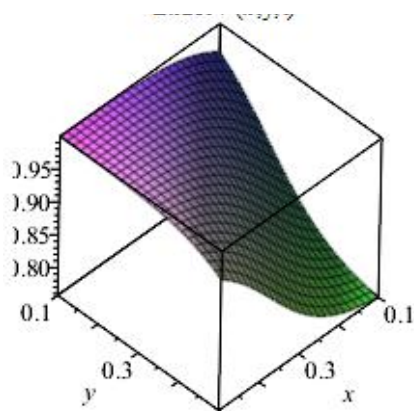

$v(x, y, t)$ exact

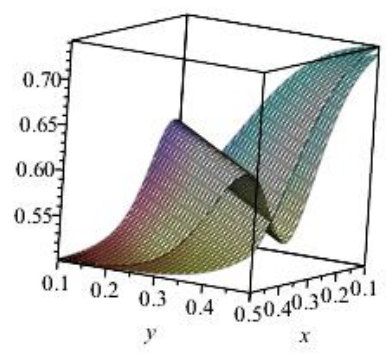

LADM and exact for $u(x, y, t)$

(d)

Figures 4. (c) and (d) show the exact and LADM of $u(x, y, t)$ and $v(x, y, t)$ for example $4,0.1 \leq x \leq 0.5 ; 0.1 \leq y \leq 0.5$; $\mathrm{t}=0.5 ; \mathrm{R}=100$

Table 2: The numerical results in comparison with the analytical solutions for various values of $x$ and $t$ for example 4 when $y=1, R=50$ and $R=100$ are respectively

\begin{tabular}{|c|c|c|c|c|c|c|c|}
\hline \multirow[b]{2}{*}{$t$} & \multirow[b]{2}{*}{$x$} & \multirow[b]{2}{*}{$\begin{array}{c}\operatorname{Exact}(u) \\
\qquad \mathrm{R}=50\end{array}$} & \multirow[b]{2}{*}{$\begin{array}{c}\operatorname{Exact}(v) \\
\mathrm{R}=100\end{array}$} & \multicolumn{4}{|c|}{ The absolute error } \\
\hline & & & & $\begin{array}{c}\operatorname{LADM}(u) \\
\mathrm{R}=50\end{array}$ & $\begin{array}{c}\operatorname{AMD}(u)[16] \\
\mathrm{R}=50\end{array}$ & $\begin{array}{c}\operatorname{LADM}(v) \\
\mathrm{R}=100\end{array}$ & $\begin{array}{c}\operatorname{AMD}(v)[16] \\
\mathrm{R}=100\end{array}$ \\
\hline \multirow{5}{*}{0.2} & 0.1 & 0.7487736486 & 0.7499939249 & $2.73 e-004$ & $7.4512 \mathrm{e}-003$ & $1.88 \mathrm{e}-006$ & $5.1579 e-006$ \\
\hline & 0.2 & 0.7477185907 & 0.7499787972 & $5.04 e-004$ & $1.3845 e-003$ & $6.55 e-006$ & $1.8001 \mathrm{e}-005$ \\
\hline & 0.3 & 0.7457712717 & 0.7499260107 & $9.20 \mathrm{e}-004$ & $2.5604 \mathrm{e}-003$ & $2.28 \mathrm{e}-005$ & $6.2817 e-005$ \\
\hline & 0.4 & 0.7422137941 & 0.7497419422 & $1.65 e-003$ & $4.6943 e-003$ & $7.96 \mathrm{e}-005$ & $2.1906 e-004$ \\
\hline & 0.5 & 0.7358362425 & 0.7491015994 & $2.84 e-003$ & $8.4719 \mathrm{e}-003$ & $2.77 e-004$ & $7.6234 \mathrm{e}-004$ \\
\hline \multirow{5}{*}{0.5} & 0.1 & 0.7480460278 & 0.7499844874 & $6.09 e-004$ & $1.7787 \mathrm{e}-003$ & $1.87 e-006$ & $1.8237 \mathrm{e}-005$ \\
\hline & 0.2 & 0.7463741051 & 0.7499458640 & $1.28 \mathrm{e}-003$ & $3.2970 \mathrm{e}-003$ & $6.53 e-006$ & 1. $6.36 \mathrm{e}-005$ \\
\hline & 0.3 & 0.7433101792 & 0.7498111486 & $2.07 e-003$ & $6.0701 \mathrm{e}-003$ & $2.28 \mathrm{e}-005$ & $2.2192 \mathrm{e}-004$ \\
\hline & 0.4 & 0.7377855554 & 0.7493420815 & $3.73 e-003$ & $1.1040 \mathrm{e}-002$ & $8.01 \mathrm{e}-005$ & $7.7219 e-004$ \\
\hline & 0.5 & 0.7281090401 & 0.7477185907 & $6.53 e-003$ & $1.9654 \mathrm{e}-002$ & $2.84 e-004$ & $2.6661 \mathrm{e}-003$ \\
\hline
\end{tabular}

The numerical results show that using of LADM by 3- components gives results more accurate than the results using 5 components by standard ADM which is presented in [16].

\section{CONCLUSION}

LADM is a powerful tool which is capable of handling coupled system of nonlinear of partial differential equations. In this paper the LADM has been successfully applied to find approximate solutions for the homogeneous form of the system of two dimensional Burger's equations without any discretization. The method presents a useful way to develop an analytic 
treatment for these systems. The proposed scheme can be applied for system more than two linear and nonlinear partial differential equations.

\section{REFERENCES}

[1] L. Debnath, Nonlinear Partial Differential Equations for Scientists and Engineers, Birkhauser, Boston, 1997.

[2] J. D. Logan, An Introduction to Nonlinear Partial Differential Equations, Wiley, New York, 1994.

[3] A. M. Wazwaz, The decomposition method applied to systems of partial differential equations and to the reactiondiffusion brusselator model, Appl. Math. Comput., 110 (2000), pp. 251-264.

[4] S. A. Khuri, A Laplace Decomposition Algorithm Applied to Class of Nonlinear Differential Equations, Jour- nal of Applied Mathematics, Vol. 1, No. 4, 2001, pp. 141- 155.

[5] H. Hosseinzadeh, H. Jafari and M. Roohani, Application of Laplace Decomposition Method for Solving Klein- Gordon Equation, World Applied Sciences Journal, Vol.8, No. 7, 2010, pp. 809-813.

[6] M. Khan, M. Hussain, H. Jafari and Y.Khan, Application of Laplace Decomposition Method to Solve Nonlinear Coupled Partial Differential Equations, World Applied Sciences Journal, Vol. 9, No. 1, 2010, pp. 13-19.

[7] E. Yusufoglu , Numerical Solution of Duffing Equation by the Laplace Decomposition Algorithm," Applied Mathematics and Computation, Vol. 177, No.2, 2006, pp. 572-580. doi:10.1016/j.amc.2005.07.072.

[8] D. Kaya and I.E. Inan, Exact and Numerical Traveling Wave Solutions for Nonlinear Coupled Equations Using Symbolic Computation, Appl. Math.and Comput. 151, (2004) 775-787.

[9] H. Jafari and V. Daftardar-Gejji, Solving Linear and Nonlinear Fractional Diffution and Wave Equations by Adomian Decomposition, Applied Mathematics and Computation, Vol. 180, No. 2, 2006 , pp. $488-497$. doi:10.1016/j.amc.2005.12.031.

[10] F. Abdelwahid, A mathematical Model of Adomian Polynomials, Applied Mathematics and Computation, Vol. 141, No. 2-3, 2003, pp. 447-453. doi:10.1016/S0096-3003(02)00266-7.

[11] Mahmoud S. Rawashdeh1 and Shehu Maitama, SOLVING COUPLED SYSTEM OF NONLINEAR PDE'S USING THE NATURAL DECOMPOSITION METHOD. International Journal of Pure and Applied Mathematics, Volume 92 No. 5 2014, 757-776.

[12] A.S. Ravi Kanth and K. Aruna, Differential transform method for solving linear and non-linear systems of partial differential equations, Phys. Lett. A., 372, 6896-6898 (2008).

[13] R. Bellman, B. G. Kashef and J. Casti, Differential Quadrature Technique for The Rapid Solution of Nonlinear Partial Differential equations, J. of Comput. Physics, 10, (1972), No.1, 40-52.

[14] H. O. AL-Humedi et.al, Modified Algorithm to Compute Adomian's Polynomial for Solving Non-Linear Systems of Partial Differential Equations, Int. J. Contemp. Math. Sciences, Vol. 5, 2010, no. 51, 2505 - 2521.

[15] M. Dehghan, H. Asgar and S. Mohammad, The Solution of Coupled Burger's Equations Using Adomian-Pade technique, Appl. Math. and Comput. 189, (2008), No 2, 1034-1047.

[16] S.M. El- Sayed and D. Kaya, On The Numerical Solution of The System of Two-dimensional Burger's Equations by The Decomposition Method, Appl. Math. and Comput. 158, (2004), 101-109. 